\title{
Rspo2 suppresses CD36-mediated apoptosis in oxidized low density lipoprotein-induced macrophages
}

\author{
HUI YAN*, SHUAI WANG ${ }^{*}$, ZHENWEI LI, ZEWEI SUN, JIE ZAN, WENTING ZHAO, \\ YANYUN PAN, ZHEN WANG, MINGJIE WU and JIANHUA ZHU \\ Department of Cardiology, First Affiliated Hospital, School of Medicine, Zhejiang University, \\ Hangzhou, Zhejiang 310009, P.R. China
}

Received August 7, 2015; Accepted June 22, 2016

DOI: $10.3892 / \mathrm{mmr} .2016 .5642$

\begin{abstract}
Oxidized low density lipoprotein (oxLDL)-induced apoptosis of macrophages contributes to the formation of atherosclerotic plaques. R-spondin 2 (Rspo2), a member of the cysteine-rich secreted proteins, has been shown to be involved in the oncogenesis of several types of cancer. It has also been found to be abundantly expressed among the four R-spondin members in macrophages. The present study was performed to determine whether Rspo2 is involved in the ox-LDL-induced apoptosis of macrophages. It was identified that Rspo 2 inhibited oxLDL-induced apoptosis in the presence of endoplasmic reticulum (ER) stress activator using flow cytometry. In addition, Rspo2 was observed to suppress oxLDL-induced ER stress and reactive oxygen species production as demonstrated by western blotting. Furthermore, analysis of the role of Rspo2 in macrophage lipid uptake identified that Rspo2 negatively regulated the Dil-oxLDL uptake by inhibiting the expression of cluster of differentiation (CD)36, through the transcription factor, peroxisome proliferator-activated receptor (PPAR) $-\gamma$. The manipulation of Rspo2 had a direct effect on PPAR- $\gamma$ nuclear translocation. In addition, chromatin immunoprecipitation analysis revealed that Rspo 2 manipulation led to regulation of the direct binding between PPAR- $\gamma$ and CD36. In conclusion, Rspo 2 was found to have a negative regulatory effect during oxLDL-induced macrophage apoptosis by regulating lipid uptake.
\end{abstract}

Correspondence to: Dr Jianhua Zhu, Department of Cardiology, First Affiliated Hospital, School of Medicine, Zhejiang University, 79 Qingchun Road, Hangzhou, Zhejiang 310009, P.R. China

E-mail: zjh_john@medmail.com.cn

*Contributed equally

Key words: oxidized low density lipoprotein, R-spondin 2, cluster of differentiation 36, peroxisome proliferator-activated receptor- $\gamma$, apoptosis

\section{Introduction}

Atherosclerosis is a chronic inflammatory disease, which is associated with several types of infiltrating inflammatory cell and contributes to mortality rates worldwide $(1,2)$. Foam cells, which are formed as a result of oxidized low-density lipoprotein (oxLDL) uptake by macrophage colony-stimulating factor- or granulocyte-macrophage colony-stimulating factor-differentiated macrophages, contribute primarily to the formation of a necrotic core in plaques and thus accelerate the development of atherosclerosis $(3,4)$. The oxLDL-induced apoptosis of macrophages is also important in the process of atherosclerosis (5). It has been shown that treatment with oxLDL at a high concentration $(100 \mu \mathrm{g} / \mathrm{ml})$ promotes macrophage apoptosis in vitro by inducing endoplasmic reticulum (ER) stress (6-9). In addition, high concentrations of oxLDL result in the activation of other signaling pathways, including c-Jun N-terminal kinase, peroxisome proliferator-activated receptor (PPAR)- $\gamma, \mathrm{p} 38$ and p53, which subsequently contribute to the apoptosis of macrophages $(7,10,11)$. This suggests that oxLDL-induced macrophage apoptosis is a complex and multi-factorial process.

R-spondins are secreted proteins, which have a thrombospondin type I repeat in their structure and are involved in activation of the Wnt signaling pathway (12). There are four members in this group, Rspo1, Rspo2, Rspo3 and Rspo4, each of which is $\sim 35 \mathrm{Kd}$ in size, and all of which have been shown to be involved in the regulation of various diseases and biological processes, including osteoporosis pseudoglioma, skeletal myogenesis, keratinocyte proliferation, mammary epithelial cell invasiveness and colorectal cancer (13-17). Preliminary data have suggested that Rspo 2 is the most abundant of the four R-spondins, which is expressed in the THP-1 macrophage-like cell line. The activation of Wnt signaling has been implicated in the protection from apoptosis in pre-adipocytes (18) and, as R-spondins have been linked with the activation of Wnt signaling, the present study hypothesized that Rspo2, due to being abundantly expressed and activating the Wnt pathway, may be involved in oxLDL-induced macrophage apoptosis. Therefore, in the present study, the monocytic THP1 cell line was used to confirm the role of Rspo2 in the ox-LDL-induced apoptosis of macrophages. 


\section{Materials and methods}

Cell culture. The THP-1 cells (American Type Culture Collection, Manassas, VA, USA) were cultured in RPMI 1640 medium (Gibco; Thermo Fisher Scientific, Inc., Waltham, MA, USA) containing 10\% fetal calf serum (Sigma-Aldrich, St. Louis, MO, USA). Prior to any treatments, the THP-1 monocytic cells were treated with phorbol myristate acetate (Sigma-Aldrich) at a concentration of $100 \mathrm{nmol} / \mathrm{l}$ for $24 \mathrm{~h}$ at $37^{\circ} \mathrm{C}$ to differentiate the cells into macrophages-like sticky cells. The differentiated macrophages $\left(1 \times 10^{6}\right)$ were treated with $40 \mu \mathrm{g} / \mathrm{ml}$ oxLDL (Guangzhou Yiyuan Biological Technology Co., Ltd., Guangzhou, China) or thapsigargin ( $\mathrm{Tg} ; 1 \mu \mathrm{M}$; Sigma-Aldrich) for $24 \mathrm{~h}$ at $37^{\circ} \mathrm{C}$ in subsequent experiments.

Transfection. According to the manufacturer's protocol, $2 \mu \mathrm{l}$ of small interfering (si)RNA (50 $\mathrm{nM}$ ) or $2 \mu \mathrm{g}$ plasmid were mixed with $5 \mu \mathrm{l}$ P3000 reagent (Life Technologies, Grand Island, NY, USA) in $100 \mu \mathrm{l}$ of opti-MEM (Gibco; Thermo Fisher Scientific, Inc.) medium. Separately, $3.75 \mu 1$ of lipo 3000 (Life Technologies) was added to another $100 \mu \mathrm{l}$ of opti-MEM medium. After $5 \mathrm{~min}$ of incubation at room temperature, the opti-MEM medium was mixed and added to $1 \times 10^{6}$ cells in a complete medium for $24 \mathrm{~h}$ at $37^{\circ} \mathrm{C}$. The siRNA knockdown or plasmid overexpression efficiencies were analyzed by detecting the mRNA levels of Rspo2.

Reverse transcription-quantitative polymerase chain reaction $(R T-q P C R)$. Total RNA was isolated using a total mRNA isolation kit (Tiangen Biotech Co., Ltd., Beijing, China), according to the manufacturer's instructions. cDNAs were generated using a PrimeScript RT reagent kit (Takara Biotechnology Co., Ltd., Dalian, China). Briefly, 500 ng total mRNA was mixed with $2 \mu 1$ PrimeScript RT reagent and added $\mathrm{ddH}_{2} \mathrm{O}$ to a total volume of $10 \mu \mathrm{l}$. The RT reaction was performed at $37^{\circ} \mathrm{C}$ for $15 \mathrm{~min}$. qPCR was conducted using SYBR-Green Premix Ex Taq (Takara Biotechnology Co., Ltd.) and detected by ABI PRISM 7500 Sequence Detection System (Applied Biosystems; Thermo Fisher Scientific, Inc.). The cycling condition were $95^{\circ} \mathrm{C}$ for $15 \mathrm{sec}$ and $60^{\circ} \mathrm{C}$ for $34 \mathrm{sec}$, for 40 cycles. The primer sequences used for Rspo 2 were: Forward, 5'-GTCTCCCTCTGAGTCCTCCC-3'; and reverse, 5'-CGTCTCCATCGGTTGCCTT-3'. Analysis of relative gene expression was perfomed using the $2^{-\Delta \Delta \mathrm{Cq}} \operatorname{method}(19)$.

Apoptosis assays. The apoptotic rates were measured using annexin V (BD Biosciences, Franklin Lakes, NJ, USA) and propidium iodide (PI; BD Biosciences,) staining kits, as previously described (20). Briefly the treated macrophage cells were incubated with annexin $\mathrm{V}$ staining for $15 \mathrm{~min}$ at room temperature, following which the PI solution was added and the cells were incubated in the dark for another $15 \mathrm{~min}$ at room temperature. Finally, the cells were analyzed using flow cytometry.

Western blotting. Following treatment, the differentiated THP-1 cells were lysed using RIPA lysis buffer (Beyotime Institute of Biotechnology, Shanghai, China) to extract the total proteins. Following extraction, $40 \mu \mathrm{g}$ protein of each sample were separated by electrophoresis on a $10 \%$ polyacrylamide SDS gel and then transferred onto a PVDF membrane (EMD
Millipore, Billerica, MA, USA). The membrane was then incubated with monoclonal rabbit anti-Rspo2 antibody (1:1,000; cat. no. ab73761; Abcam, Cambridge, MA, USA), monoclonal rabbit anti-GAPDH antibody (1:1,000; cat. no. AG019; Beyotime Institute of Biotechnology, Beijing, China), monoclonal mouse anti-protein kinase RNA-like ER kinase (PERK) antibody (1:1,000; cat. no. 12185; Cell Signaling Technology, Inc., Danvers, MA, USA), monoclonal rabbit anti lectin-like LDL receptor-1 (LOX-1) antibody (1:1,000; cat. no. ab60178; Abcam), monoclonal mouse anti-scavenger receptor A (SRA) antibody (1:1,000; cat. no. ab36625; Abcam), rabbit anti-CD36 antibody (1:1,000; cat. no. ab78054; Abcam) and monoclonal rabbit anti-PPAR- $\gamma$ antibody $(1: 1,000$; cat. no. 2435; Cell Signaling Technology, Inc.) at $4^{\circ} \mathrm{C}$ overnight. The next day, the membranes were washed using Tris-buffered saline/Tween (TBST) for $15 \mathrm{~min}$, and further incubated with goat anti-rabbit (cat. no. CW0103M) or goat anti-mouse (cat. no. CW0102M) specific antibodies (1:5,000; Beijing Kangwei Biological Technology Co., Ltd., Beijing, China) for $1 \mathrm{~h}$ at room temperature. Finally, following washing with TBST, the protein signals were detected using ECL reagent (Pierce, Bonn, Germany). Quantification of western blot analysis was performed using Image $\mathrm{J} 2 \mathrm{x}$ software (National Institutes of Health, Bethesda, MD, USA).

ROS measurement. ROS measurement was performed using 2,7-dichlorofluorescein diacetate dye, according to the manufacturer's protocol (Beyotime Institute of Biotechnology). The cells were incubated with this dye $(10 \mu \mathrm{M})$ for $30 \mathrm{~min}$ to enable its uptake by the cells, following which the cells were washed with phosphate-buffered saline (PBS) three times. The dye inside the cells emitted a fluorescent signal on meeting free radicals, and images of the fluorescent signal were captured using an Olympus fluorescence microscope (Olympus Corporation, Tokyo, Japan).

Detection of liquid uptake. For examining Dil-oxLDL uptake, the cells were washed twice with RPMI 1640 medium and then incubated with Dil-oxLDL ( $10 \mu \mathrm{g} / \mathrm{ml}$; Guangzhou Yiyuan Biological Technology Co., Ltd.) for $3 \mathrm{~h}$ at $37^{\circ} \mathrm{C}$ in the dark, according to the manufacturer's instructions. Subsequently, the cells were washed with PBS for $15 \mathrm{~min}$ in the dark and were observed under an Olympus fluorescence microscope.

Immunofluorescence staining. Following treatment, the cultured cells were fixed with $4 \%$ paraformaldehyde solution and then permeabilized with $0.5 \%$ TritonX-100 solution. The cells were then incubated with monoclonal rabbit anti-PPAR- $\gamma$ antibody (1:100; cat. no. 2435; Cell Signaling Technology, Inc.) at $37^{\circ} \mathrm{C}$ for $2 \mathrm{~h}$ and washed with PBS. This was followed by incubation with goat anti-rabbit secondary antibody (1:200; cat. no. CW0103M; Beijing Kangwei Biological Technology Co., Ltd.) for another $1 \mathrm{~h}$ at $37^{\circ} \mathrm{C}$. The cells were then incubated with DAPI (Guangzhou Yiyuan Biological Technology Co., Ltd.), and were observed under a Zeiss confocal microscope (Carl Zeiss, Inc., Oberkochen, Germany) for visualization of the staining signal.

Chromatin immunoprecipitation (ChIP) assay. ChIP assays were performed using a kit (Cell Signaling Technology, Inc.), as described previously (21). In brief, the cells were 


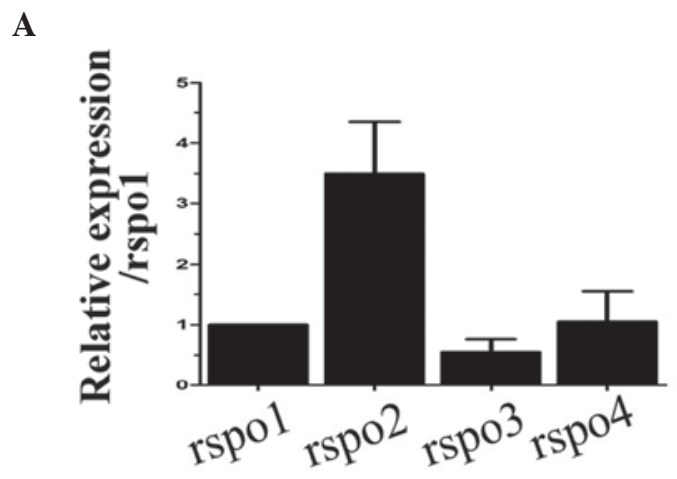

B

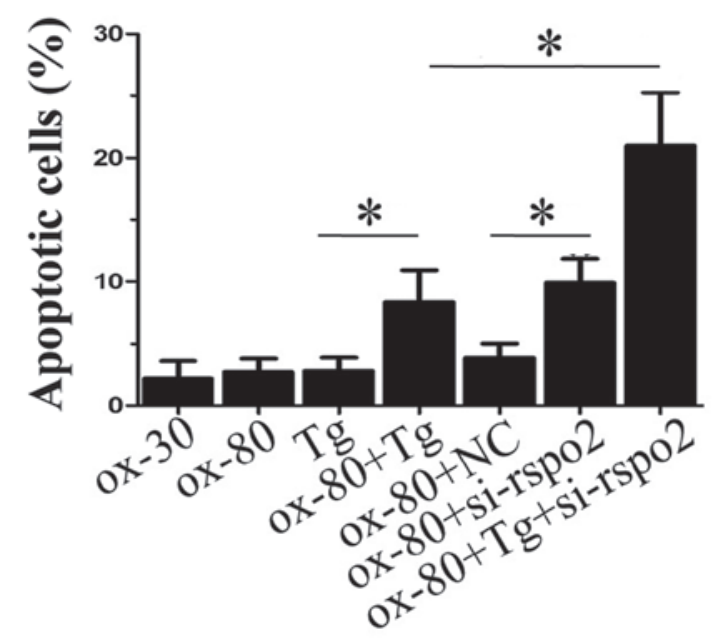

C

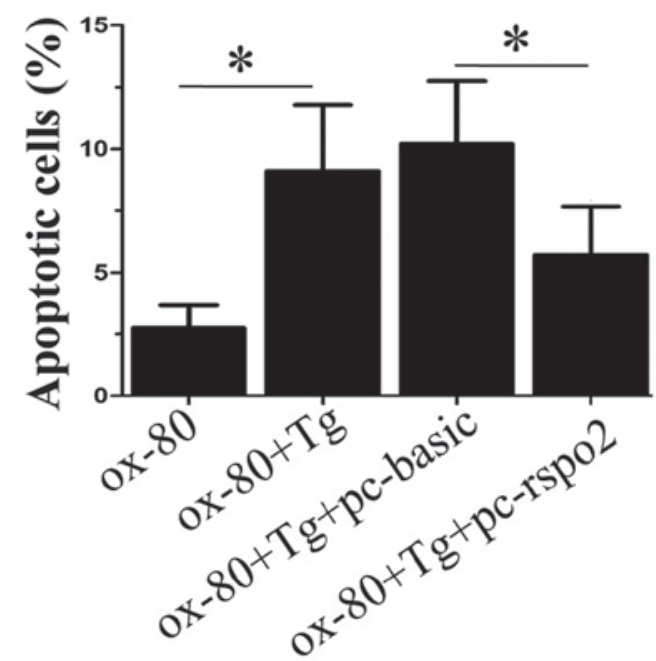

Figure 1. Rspo2 negatively regulates oxLDL-induced THP-1 cell apoptosis under ER stress. (A) THP-1 cells were analyzed for the expression of Rspo1, Rspo2, Rspo3 and Rspo4 using reverse transcription-polymerase chain reaction analysis. The expression of 18S, a ribosomal gene, was used as an endogenous control for normalization. (B) THP-1 cells, either alone or transfected with NC or Rspo2 siRNA, were incubated for $24 \mathrm{~h}$ with the indicated concentrations of oxLDL alone or in combination with Tg. Subsequently, apoptosis was assessed and the percentage of apoptotic cells were determined. (C) THP-1 cells alone or overexpressing either the vector (pc-basic) or Rspo2 plasmid (pc-rspo2), were incubated for $24 \mathrm{~h}$ with oxLDL ( $80 \mu \mathrm{g} / \mathrm{ml}$ ) alone or in combination with Tg. The apoptotic rate was assessed and presented as a bar graph. Values are expressed as the mean \pm standard deviation. " $\mathrm{P}<0.05$, comparison indicated by brackets. siRNA, small interfering RNA; oxLDL, oxidized low density lipoprotein; Rspo, R-spondin; Tg, thapsigargin.

harvested and fixed using 4\% formaldehyde for $10 \mathrm{~min}$. Following chromosome shearing with nuclease, smaller DNA fragments were obtained. Following this, the PPAR- $\gamma$ protein was immunoprecipitated using rabbit anti-PPAR- $\gamma$ antibody and the complex was incubated with $\mathrm{NaCl}(5 \mathrm{M})$ solution at $65^{\circ} \mathrm{C}$ to reverse crosslinking, following which the DNA was purified using a DNA purification kit (Cell Signaling Technology, Inc.). The purified DNA was used for PCR (ABI PRISM 7500 sequence detection system; Applied Biosystems; Thermo Fisher Scientific, Inc.) to detect the relative expression of target fragments. SYBR Premix Ex Taq (Takara Biotechnology Co., Ltd.) was used to amplify the DNA $(500 \mathrm{ng})$ with incubation at $95^{\circ} \mathrm{C}$ for $30 \mathrm{sec}$, and 40 cycles of $95^{\circ} \mathrm{C}$ for $5 \mathrm{sec}$ and $60^{\circ} \mathrm{C}$ for $30 \mathrm{sec}$. The results were quantified using the $2^{-\Delta \Delta \mathrm{Cq}}$ method. The specific primers used to amplify the binding site of PPAR- $\gamma$ to the CD36 promoter were as follows: Forward 5'-GCGATATCGAGT TATTCCG-3' and reverse 3'-ACTACAGGTGTGCGCCAC CATG-5'.

Statistical analysis. The statistical significance of differences between groups were calculated with unpaired $t$-tests using
SPSS software (version 17; SPSS, Inc., Chicago, IL, USA). The values are expressed as the mean \pm standard deviation. $\mathrm{P}<0.05$ was considered to indicate a statistically significant difference.

\section{Results}

Rspo2 inhibits oxLDL-induced apoptosis under ER stress in macrophages. The present study first compared the expression of four R-spondinsin THP-1 cells using qPCR. As shown in Fig. 1A, the mRNA expression of Rspo2 was the highest among all the R-spondins. To identify whether the high expression level of Rspo2 had any functional role in the oxLDL-induced apoptosis of macrophages, the expression of Rspo2 was simultaneously inhibited by siRNA and overexpressed by ectopic expression, and the apoptotic rates were assessed. As shown in Fig. 1B, neither treatment with oxLDL at two concentrations or with the ER stress activator, $\mathrm{Tg}$, alone resulted in the induction of macrophages apoptosis. However, the combined treatment of the differentiated THP-1 cells with Tg and oxLDL significantly induced apoptosis. Notably, following Rspo2 ablation by siRNA in the differentiated THP-1 cells, oxLDL treatment alone was able to induce the apoptosis of macrophages. In 
A
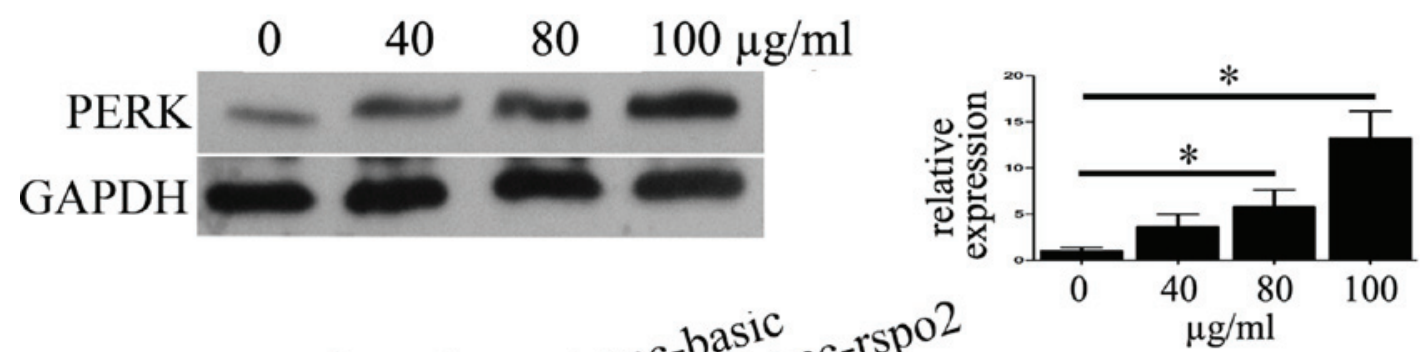

B

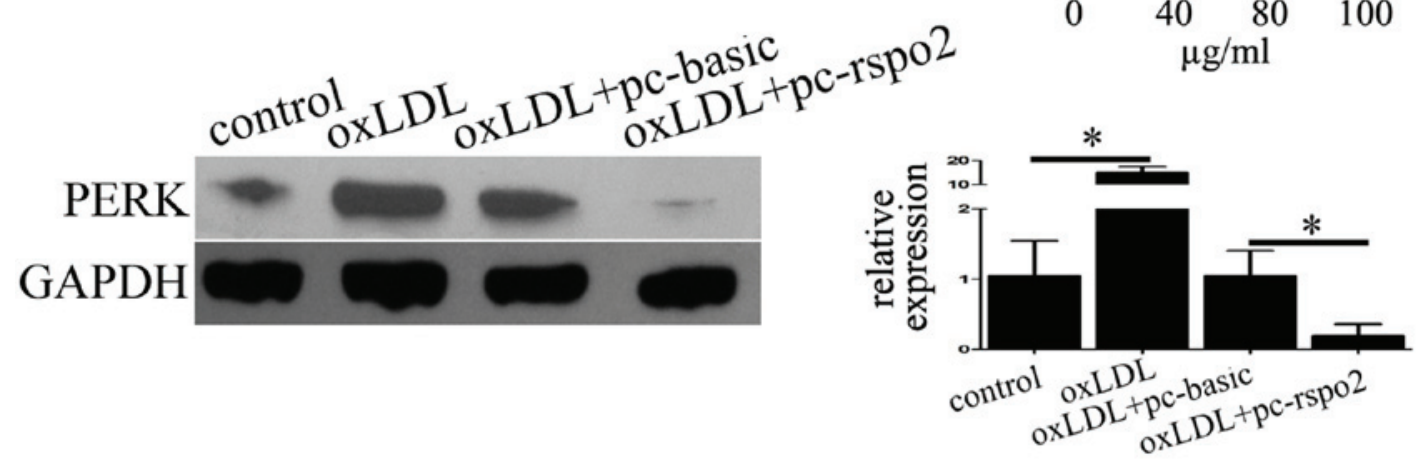

C

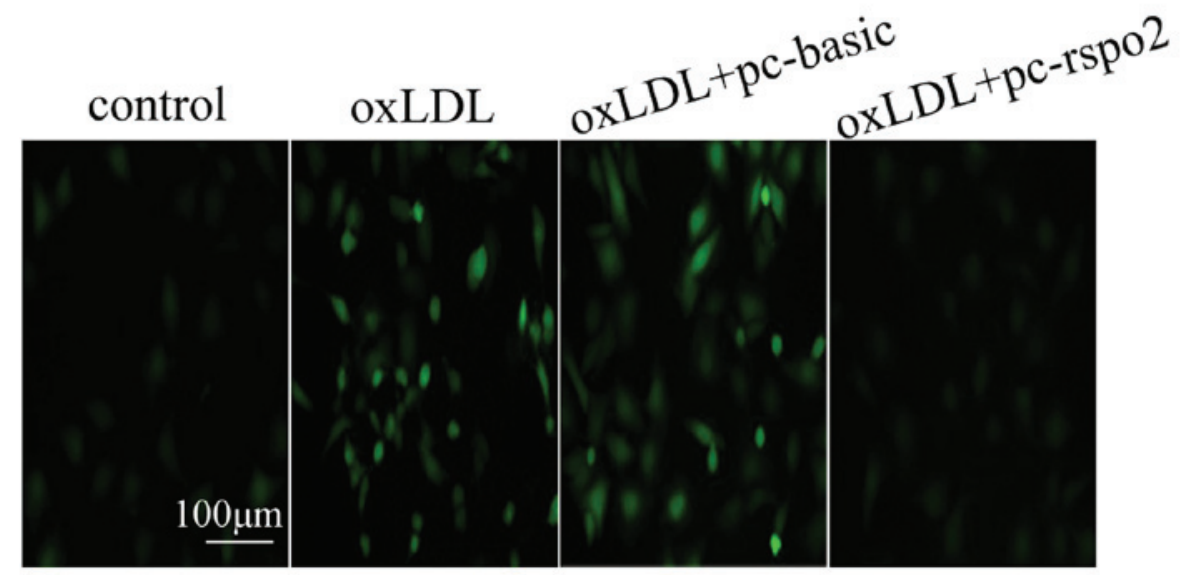

Figure 2. Rspo2 suppresses oxLDL-induced ER stress and ROS production. (A) Expression of the ER stress marker, PERK, was assessed using western blotting in THP-1 cells incubated with the indicated concentrations of oxLDL $(0-100 \mu \mathrm{g} / \mathrm{ml})$ for $24 \mathrm{~h}$. The expression of GAPDH was used as a loading control. (B) Expression of PERK was assessed in THP-1 cells alone or in those transfected with pc-basic or pc-rspo2, and treated with or without oxLDL $(100 \mu \mathrm{g} / \mathrm{ml})$ for $24 \mathrm{~h}$. The expression of GAPDH served as a loading control. (C) THP-1 cells transfected with pc-basic or pc-rspo2 and treated with or without oxLDL $(100 \mu \mathrm{g} / \mathrm{ml})$ for $24 \mathrm{~h}$, were labeled with 2,7-dichlorofluorescein diacetate dye to assess the production of ROS. The labeled cells produced fluorescent signal proportional to the ROS levels, and this signal was recorded using an Olympus fluorescence microscope. "P<0.05, comparison indicated by brackets. oxLDL, oxidized low density lipoprotein; Rspo, R-spondin; ER, endoplasmic reticulum; ROS, reactive oxygen species; PERK, protein kinase RNA-like ER kinase; siRNA, small interfering RNA.

addition, when the Rspo2-ablated THP-1 cells were treated with a combination of oxLDL and Tg, an additional increase in the rate of macrophage apoptosis was observed. Furthermore, the ectopic overexpression of Rspo2 was able to reverse the induction of apoptosis by oxLDL and $\mathrm{Tg}$ in the differentiated THP-1 cells, as shown in Fig. 1C.

Rspo2 suppresses ER stress and ROS production in oxLDL treatment. As it was observed that oxLDL only induced macrophage apoptosis in the presence of $\mathrm{Tg}$, which increases ER stress, or under Rspo2 ablation, the present study investigated whether the expression of Rspo2 is involved in modulating ER stress. The protein expression of PERK, which is described as an ER stress marker (22), was determined by treating the THP-1 cells with increasing concentrations of oxLDL. As shown in Fig. 2A, the expression of PERK increased in a dose-dependent manner with increasing concentrations of oxLDL. However, the ectopic expression of Rspo2 in THP-1 cells treated with oxLDL, resulted in a significant reduction in the expression of PERK (Fig. 2B). These results suggested that the overexpression of Rspo2 inhibited ER stress. The present study also measured the production of ROS in the THP-1 cells. As shown in Fig. 2C, the data revealed that oxLDL treatment induced ROS production, shown as increased fluorescent signal under the microscope. Similarly, the overexpression of Rspo2 significantly reduced this signal, thus supporting the hypothesis that Rspo2 contributes to reductions in ER stress and ROS production.

Rspo2 negatively regulates lipid uptake and the expression of scavenger receptor, CD36. As oxLDL-induced macrophage apoptosis is a later process and is preceded by its uptake by macrophages, the present study investigated whether Rspo 2 is 
A

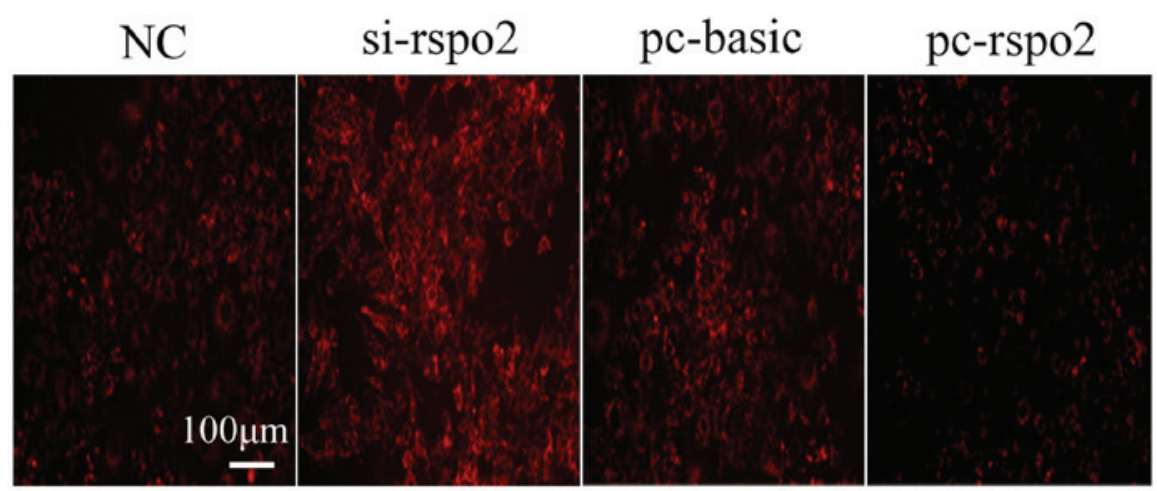

B
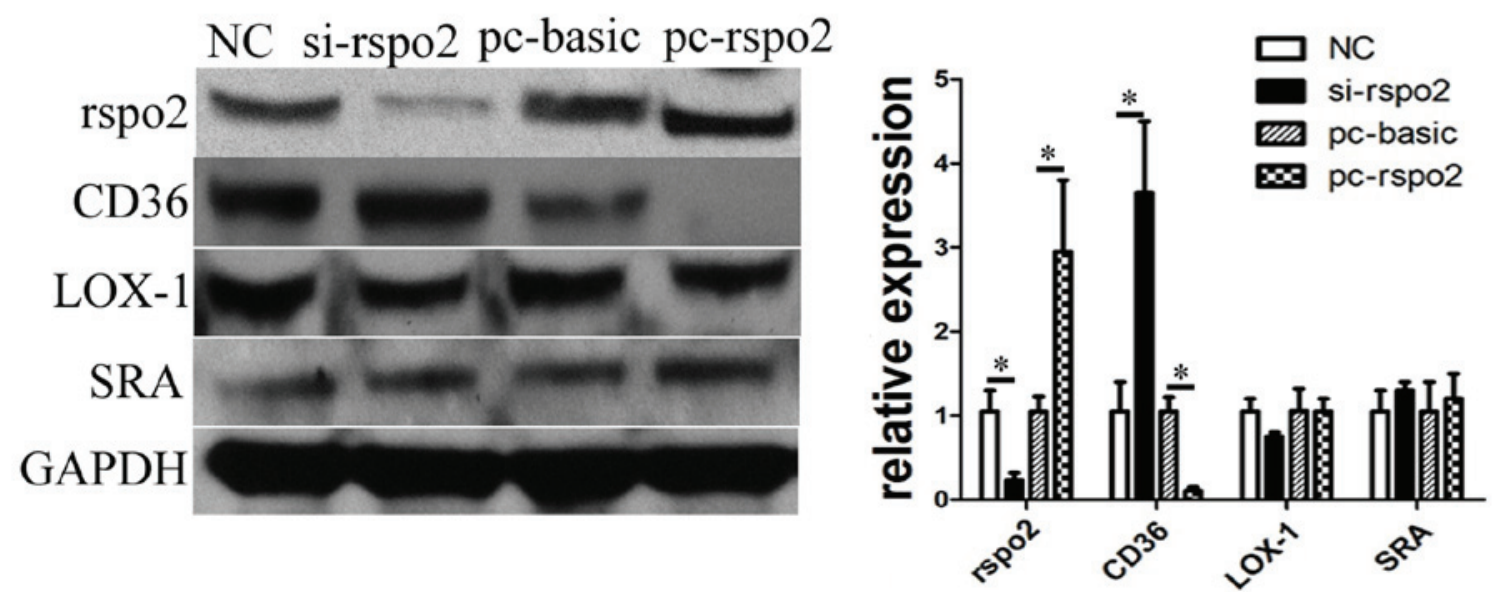

Figure 3. Rspo2 negatively regulates lipid uptake and the expression of CD36. (A) THP-1 cells were transfected with pc-basic, pc-rspo2, NC, or si-rspo2 for $24 \mathrm{~h}$. The cells from the different transfection conditions were then treated with Dil-oxLDL (10 $\mu \mathrm{g} / \mathrm{ml})$ for $3 \mathrm{~h}$, following which the fluorescent signal was recorded under a microscope. (B) THP-1 cells were transfected with NC, si-rspo2, pc-basic or pc-rspo2 for $24 \mathrm{~h}$. The total proteins extracted from these different groups were blotted to detect the protein expression of Rspo2, CD36, LOX-1 and SRA. The expression of GAPDH was used as a loading control. "P<0.05, comparison indicated by brackets. Rspo, R-spondin; pc-basic, empty plasmid pCMV; pc-rspo2, Rspo2 plasmid; si-rspo2, Rspo2-specific siRNA; NC, negative control; CD36, cluster of differentiation 36; LOX-1, lectin-like LDL receptor-1; SRA, scavenger receptor A.

involved in oxLDL uptake. To determine this, the differentiated THP-1 cells with Rspo2 ablation or overexpression were treated with Dil-oxLDL. It was observed that the Rspo2-ablated cells had increased uptake of Dil-oxLDL, whereas the cells overexpressing Rspo 2 had markedly lower levels of lipid uptake, as shown in Fig. 3A. The present study also analyzed the regulation of three types of scavenger receptor, CD36, SRA and LOX-1, which are predominantly involved in lipid uptake by macrophages (23). Of note, it was observed that Rspo2 negatively regulated the protein expression of CD36, but had no effect on the expression levels of SRA or LOX-1, as shown in Fig. 3B. The overexpression of Rspo2 inhibited the expression of CD36, whereas the ablation of Rspo2 marginally increased the expression of CD36, compared with the respective controls.

Rspo2 decreases the expression of CD36 through inhibiting $P P A R-\gamma$. In order to understand how Rspo 2 mediates the regulation of the expression of CD36, the present study investigated the transcription factor, PPAR- $\gamma$, which has been previously demonstrated to regulate the expression of CD36 in macrophages (24). The expression of PPAR- $\gamma$ was analyzed in THP- 1 cells transfected with either the vector or Rspo 2 plasmid and treated with oxLDL. As shown in Fig. 4A, the Rspo2-overexpressing cells treated with oxLDL had reduced expression of PPAR- $\gamma$. This was consistent with the above-mentioned reduced expression of CD36 in the Rspo2-overexpressing cells. The nuclear translocation of PPAR- $\gamma$ was further examined by analyzing its expression using immunoflorescence staining. It was observed that oxLDL led to the translocation of PPAR- $\gamma$ into the nucleus, however, the overexpression of Rspo2 reduced its nuclear translocation in THP-1 cells, as seen in Fig. 4B. Finally, the binding activity of PPAR $-\gamma$ to the CD36 promoter was analyzed by ChIP analysis. As seen in Fig. 4C, the binding activity of PPAR- $\gamma$ and CD36 increased following oxLDL treatment, but was significantly reduced in the cells overexpressing Rspo2. This suggested that PPAR- $\gamma$ was bound to the CD36 promoter in the presence of ox-LDL, and that the overexpression of Rspo 2 somehow reduced its binding to the CD36 promoter, and thus reduced its expression.

\section{Discussion}

The present study was performed to understand the role of Rspo 2 in the oxLDL-induced apoptosis of macrophages. 
$\mathbf{A}$
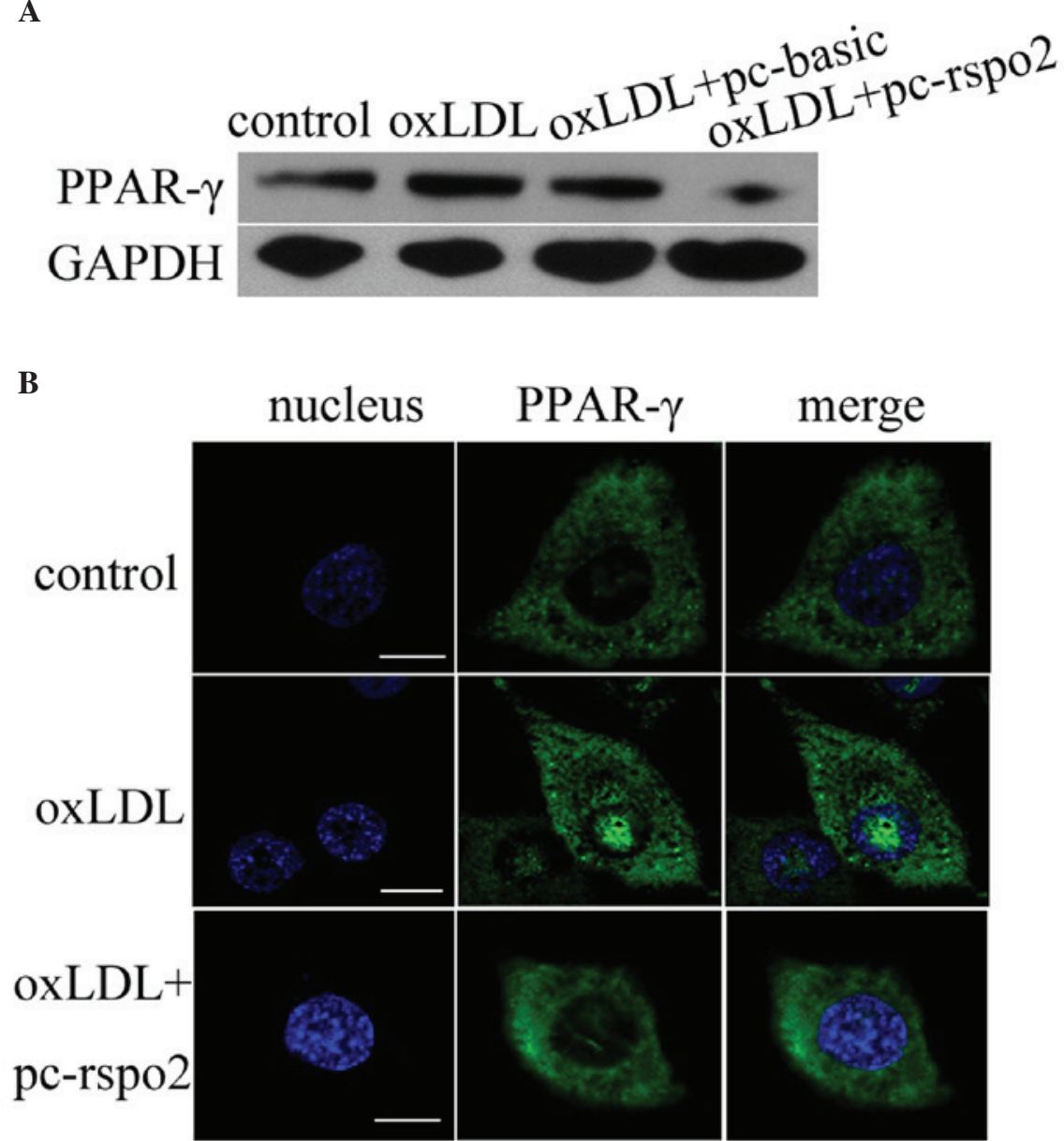

$\mathbf{C}$

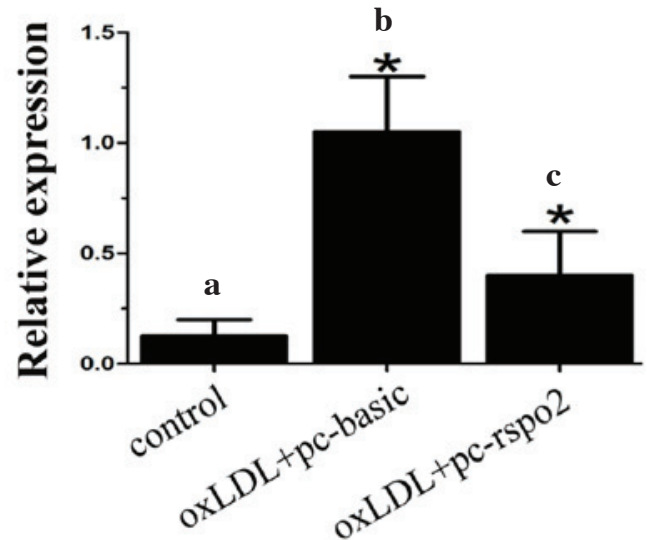

Figure 4. Rspo2 inhibits the expression of PPAR- $\gamma$, and the nuclear translocation and transcriptional activity of PPAR- $\gamma$. (A) Expression of PPAR- $\gamma$ was assessed in THP-1 cells alone or transfected with pc-basic or pc-rspo2, and treated with or without oxLDL (100 $\mu \mathrm{g} / \mathrm{ml})$ for $24 \mathrm{~h}$. GAPDH served as a loading control. (B) THP-1 cells alone or transfected with pc-rspo2 were treated with or without oxLDL $(100 \mu \mathrm{g} / \mathrm{ml})$ for $24 \mathrm{~h}$. PPAR- $\gamma$ immunostaining was then performed to assess its localization and images were captured under a Zeiss confocal microscope. (C) THP-1 cells (a) alone, (b) transfected with pc-basic or (c) transfected with pc-rspo2, were treated with oxLDL $(100 \mu \mathrm{g} / \mathrm{ml})$ for $24 \mathrm{~h}$. Chromatin immunoprecipitation assays were performed using antibodies against PPAR- $\gamma$ and the expression of CD36 was analyzed using polymerase chain reaction analysis. Total chromatin gathered prior to immunoprecipitation served as an input control. Values are expressed as the mean \pm standard deviation. $\mathrm{P}<0.05$, comparison indicated by brackets. PPAR- $\gamma$, peroxisome proliferator-activated receptor; Rspo, R-spondin; pc-basic, empty plasmid pCMV; pc-rspo2, Rspo2 plasmid; si-rspo2, Rspo2-specific siRNA; NC, negative control; CD36, cluster of differentiation 36 .

It was demonstrated that Rspo2 had a functional role in oxLDL-mediated macrophage apoptosis and is the first report, to the best of our knowledge, linking Rspo2 to the regulation of macrophage biology, particularly apoptosis. In another study, Rspo 2 knockdown has been linked with reduced cell viability of human Schwann tumors, however, 
this involved the regulation of Wnt signaling (25). Until now, the majority of studies have suggested that the functional activity of $\mathrm{R}$-spondin proteins has been via the regulation of Wnt signaling in all biological pathways, including cancer $(16,26)$. However, the present study is the first to establish the role of $\mathrm{R}$-spondin proteins in the regulation of atherosclerosis.

According to previous literature, lipid uptake in macrophages is a key factor during the process of cardiovascular disease (5). Of note, the present study found that Rspo2 protein negatively regulated the uptake of oxLDL by macrophages and that this was mediated through regulation of the expression of the scavenger receptor protein, CD36, which is involved in liquid uptake by macrophages. The effect of Rspo2 on CD36 was mediated through regulation of the nuclear translocation of PPAR- $\gamma$. This particular observation has an implication in preventing oxLDL uptake by macrophages if Rspo2 is overexpressed, ultimately leading to the inhibition of foam cell formation and finally reducing the overall ability of necrotic plaque formation in atherosclerosis.

The scavenger receptor, CD36, is predominantly distributed on different immune cell subsets, including macrophages and dendritic cells, and other cell types, including platelets, endothelial cells, adipocytes and muscle cells, and has been shown to be important in fatty acid metabolism (27), heart disease (28) and the processing of dietary fat (29). Thus, it has implications in glucose tolerance, hypertension, diabetes, cardiovascular problems, Alzheimer's disease and lipoprotein endocytosis in macrophages $(23,30)$. In this context, the data obtained in the present study, which suggested that Rspo2 has the ability to regulate the expression of CD36, indicates the potential of Rspo 2 as a suitable target for other diseases, which requires investigation.

Our previous study showed that Wnt pathway positively regulated the expression of CD36 (21), whereas Rspo2, a recognized Wnt pathway activator, was observed to inhibit the expression of CD36. At present, this differential effect remains to be fully elucidated, however, it may be that Rspo2 inhibited PPAR- $\gamma$ nuclear translocation through another mechanism in the macrophages. In addition, there are further questions remaining, including whether ox LDL can also regulate the expression of Rspo2, and whether Rspo2 is involved in the regulation of liquid outflow from the macrophages. Further investigations are required to address these questions of interest.

In conclusion, the present study showed that the Rspo2 protein protected against the oxLDL-induced apoptosis of macrophages through negative regulation of the expression of the scavenger receptor protein, CD36. In addition Rspo2 decreased oxLDL-induced ER stress and ROS production in the differentiated macrophages. The findings of the current study provide novel insight on lipid overload-associated disease, including atherosclerosis, and may be aid the development of improved therapies.

\section{Acknowledgements}

This work was funded by the Natural Science Foundation of China (grant nos. 81200214/H0215 81400205 and LQ14H020001).

\section{References}

1. Braunwald E: Shattuck lecture-cardiovascular medicine at the turn of the millennium: Triumphs, concerns and opportunities. N Engl J Med 337: 1360-1369, 1997.

2. Lloyd-Jones D, Adams RJ, Brown TM, Carnethon M, Dai S, De Simone G, Ferguson TB, Ford E, Furie K, Gillespie C, et al: Executive summary: Heart disease and stroke statistics-2010 update: A report from the American Heart Association. Circulation 121: 948-954, 2010.

3. Libby P, Ridker PM and Maseri A: Inflammation and atherosclerosis. Circulation 105: 1135-1143, 2002.

4. Waldo SW, Li Y, Buono C, Zhao B, Billings EM, Chang J and Kruth HS: Heterogeneity of human macrophages in culture and in atherosclerotic plaques. Am J Pathol 172: 1112-1126, 2008.

5. Moore KJ and Tabas I: Macrophages in the pathogenesis of atherosclerosis. Cell 145: 341-355, 2011.

6. Chang YC, Huang KX, Huang AC, Ho YC and Wang CJ: Hibiscus anthocyanins-rich extract inhibited LDL oxidation and oxLDL-mediated macrophages apoptosis. Food Chem Toxicol 44: 1015-1023, 2006.

7. Deigner HP, Claus R, Bonaterra GA, Gehrke C, Bibak N, Blaess M, Cantz M, Metz J and Kinscherf R: Ceramide induces aSMase expression: Implications for ox LDL-induced apoptosis, FASEB J 15: 807-814, 2001.

8. Seimon TA, Nadolski MJ, Liao X, Magallon J, Nguyen M, Feric NT, Koschinsky ML, Harkewicz R, Witztum JL, Tsimikas S, et al: Atherogenic lipids and lipoproteins trigger CD36-TLR2-dependent apoptosis in macrophages undergoing endoplasmic reticulum stress. Cell Metab 12: 467-482, 2010.

9. Erbay E, Babaev VR, Mayers JR, Makowski L, Charles KN, Snitow ME, Fazio S, Wiest MM, Watkins SM, Linton MF and Hotamisligil GS: Reducing endoplasmic reticulum stress through a macrophage lipid chaperone alleviates atherosclerosis. Nat Med 15: 1383-1391, 2009.

10. Oh J, Riek AE, Weng S, Petty M, Kim D, Colonna M, Cella M and Bernal-Mizrachi C: Endoplasmic reticulum stress controls M2 macrophage differentiation and foam cell formation. J Biol Chem 287: 11629-11641, 2012.

11. Giovannini C, Varì R, Scazzocchio B, Sanchez M, Santangelo C, Filesi C, D'Archivio M and Masella R: OxLDL induced p53-dependent apoptosis by activating p38MAPK and PKC $\delta$ signaling pathways in J774A.1 macrophage cells. J Mol Cell Biol 3: 316-318, 2011.

12. Yoon JK and Lee JS: Cellular signaling and biological functions of R-spondins. Cell Signal 24: 369-377, 2012.

13. Han XH, Jin YR, Seto M and Yoon JK: A WNT/beta-catenin signaling activator, $\mathrm{R}$-spondin, plays positive regulatory roles during skeletal myogenesis. J Biol Chem 286: 10649-10659, 2011.

14. Boyden LM, Mao J, Belsky J, Mitzner L, Farhi A, Mitnick MA, Wu D, Insogna K and Lifton RP: High bone density due to a mutation in LDL-receptor-related protein 5. N Engl J Med 346: 1513-1521, 2002.

15. Chua AW, Ma D, Gan SU, Fu Z, Han HC, Song C, Sabapathy K and Phan TT: The role of R-spondin 2 in keratinocyte proliferation and epidermal thickening in keloid scarring. J Invest Dermatol 131: 644-654, 2011.

16. Wu C, Qiu S, Lu L, Zou J, Li WF, Wang O, Zhao H, Wang H, Tang J, Chen L, et al: RSPO2-LGR5 signaling has tumour-suppressive activity in colorectal cancer. Nat Commun 5: 3149, 2014.

17. Klauzinska M, Baljinnyam B, Raafat A, Rodriguez-Canales J, Strizzi L, Greer YE, Rubin JS and Callahan R: Rspo2/Int7 regulates invasiveness and tumorigenic properties of mammary epithelial cells. J Cell Physiol 227: 1960-1971, 2012.

18. Longo KA, Kennell JA, Ochocinska MJ, Ross SE, Wright WS and MacDougald OA: Wnt signaling protects 3T3-L1 preadipocytes from apoptosis through induction of insulin-like growth factors. J Biol Chem 277: 38239-38244, 2002.

19. Livak KJ and Schmittgen TD: Analysis of relative gene expression data using real-time quantitative PCR and the 2(-Delta Delta C(T)) Method. Methods 25: 402-408, 2001.

20. Devries-Seimon T, Li Y, Yao PM, Stone E, Wang Y, Davis RJ, Flavell R and Tabas I: Cholesterol-induced macrophage apoptosis requires ER stress pathways and engagement of the type A scavenger receptor. J Cell Biol 171: 61-73, 2005. 
21. Wang S, Sun Z, Zhang X, Li Z, Wu M, Zhao W, Wang H, Chen T, Yan $\mathrm{H}$ and Zhu J: Wnt 1 positively regulates CD36 expression via TCF4 and PPAR- $\gamma$ in macrophages. Cell Physiol Biochem 35: 1289-1302, 2015.

22. Liu J, Lee J, Salazar Hernandez MA, Mazitschek R and Ozcan U: Treatment of obesity with celastrol. Cell 161: 999-1011, 2015.

23. Murphy JE, Tedbury PR, Homer-Vanniasinkam S, Walker JH and Ponnambalam S: Biochemistry and cell biology of mammalian scavenger receptors. Atherosclerosis 182: 1-15, 2005.

24. Tontonoz P, Nagy L, Alvarez JG, Thomazy VA and Evans RM: PPARgamma promotes monocyte/macrophage differentiation and uptake of oxidized LDL. Cell 93: 241-252, 1998.

25. Watson AL, Rahrmann EP, Moriarity BS, Choi K, Conboy CB Greeley AD, Halfond AL, Anderson LK, Wahl BR, Keng VW, et al: Canonical Wnt/ $\beta$-catenin signaling drives human schwann cell transformation, progression and tumor maintenance. Cancer Discov 3: 674-689, 2013.
26. Chartier C, Raval J, Axelrod F, Bond C, Cain J, Dee-Hoskins C, Ma S, Fischer MM, Shah J, Wei J, et al: Therapeutic targeting of tumor-derived R-spondin attenuates $\beta$-catenin signaling and tumorigenesis in multiple cancer types. Cancer Res 76: 713-723, 2016.

27. Hajri T, Han XX, Bonen A and Abumrad NA: Defective fatty acid uptake modulates insulin responsiveness and metabolic responses to diet in CD36-null mice. J Clin Invest 109: 1381-1389, 2002.

28. Febbraio M, Podrez EA, Smith JD, Hajjar DP, Hazen SL, Hoff HF, Sharma K and Silverstein RL: Targeted disruption of the class B scavenger receptor CD36 protects against atherosclerotic lesion development in mice. J Clin Invest 105: 1049-1056, 2000.

29. Drover VA, Ajmal M, Nassir F, Davidson NO, Nauli AM, Sahoo D, Tso P and Abumrad NA: CD36 deficiency impairs intestinal lipid secretion and clearance of chylomicrons from the blood. J Clin Invest 115: 1290-1297, 2005.

30. Rać ME, Safranow K and Poncyljusz W: Molecular basis of human CD36 gene mutations. Mol Med 13: 288-296, 2007. 